\title{
The role of near-infrared spectroscopy in the detection of vulnerable atherosclerotic plaques
}

Martin Horvath, Petr Hajek, Cyril Stechovsky, Jakub Honek, Miloslav Spacek, Josef Veselka

Department of Cardiology, $2^{\text {nd }}$ Medical School, Charles University, University Hospital Motol and $2^{\text {nd }}$ Medical School, Charles University, Prague, Czech Republic

Submitted: 6 November 2014

Accepted: 20 April 2015

Arch Med Sci 2016; 12, 6: 1308-1316

DOI: 10.5114/aoms.2016.62904

Copyright $\odot 2016$ Termedia \& Banach

\section{Abstract}

Coronary artery disease is the leading cause of mortality worldwide. Most acute coronary syndromes are caused by a rupture of a vulnerable atherosclerotic plaque which can be characterized by a lipid-rich necrotic core with an overlying thin fibrous cap. Many vulnerable plaques can cause angiographically mild stenoses due to positive remodelling, which is why the extent of coronary artery disease may be seriously underestimated. In recent years, we have witnessed a paradigm shift in interventional cardiology. We no longer focus solely on the degree of stenosis; rather, we seek to determine the true extent of atherosclerotic disease. We seek to identify high-risk plaques for improvement in risk stratification of patients and prevention. Several imaging methods have been developed for this purpose. Intracoronary near-infrared spectroscopy is one of the most promising. Here, we discuss the possible applications of this diagnostic method and provide a comprehensive overview of the current knowledge.

Key words: near-infrared spectroscopy, lipid-core plaque, vulnerable plaque.

\section{Introduction}

Coronary artery disease (CAD) is the leading cause of morbidity and mortality worldwide [1, 2]. It is known that most acute coronary syndromes (ACS) are caused by a rupture and subsequent thrombosis of an atherosclerotic plaque [3]. The term vulnerable atherosclerotic plaque (VP) was established in 1989 [4] to functionally define a lesion with a potential of rupture. Since then, much attention has been devoted to revealing the anatomical nature of VP. The goal is to identify VPs in vivo to prevent their rupture and thereby avert myocardial infarction and sudden cardiac death.

Autopsy studies have revealed that the most common non-thrombosed lesion that most resembles a ruptured plaque is the thin cap fibroatheroma (TCFA) [5]. This type of lesion is characterized by active inflammation and a large necrotic core of lipid and cellular debris with an overlying thin fibrous cap (measuring $<65 \mu \mathrm{m}$ ) that contains many macrophages and only a few smooth muscle cells [5]. Other lesions that are associated with acute coronary syndrome include plaque erosion (30-35\% of cases) and calcified nodules (about 5\%) [6]. This review deals only with the detection of TCFA. A number of diagnostic imaging

\author{
Corresponding author: \\ Martin Horvath MD \\ Department of Cardiology \\ $2^{\text {nd }}$ Medical School \\ Charles University \\ University Hospital Motol \\ $2^{\text {nd }}$ Medical School \\ Charles University \\ V Úvalu 84 \\ 15006 Prague \\ Czech Republic \\ Phone: +42 0737736516 \\ E-mail: martin@horvath.cz
}


methods and numerous biomarkers for the identification of high-risk plaques in vivo have been tested [7-24].

Some of the imaging methods focus on the microanatomy of an atherosclerotic plaque [6-8]. Among the non-invasive methods, computed tomography (CT) coronary angiography (CTA) seems to exhibit the greatest potential. Some characteristics of atherosclerotic plaques detected by CTA (the presence of positive remodelling, low attenuation plaque and spotty calcification) are associated with the culprit lesions of ACS [9]. The invasive methods include intravascular ultrasound (IVUS), IVUS virtual histology and intravascular optical coherence tomography (OCT). Intravascular ultrasound is the method with the most solid evidence in VP detection, as only IVUS has large prospective studies that have proven its ability to identify lesions that cause future coronary events $[10,11]$. Interestingly, these lesions were often angiographically mild and frequently harboured thin-cap fibroatheromas with a large plaque burden due to positive remodelling of the vessel [10]. A large plaque burden has recently been linked to the presence of lipid cores in lesions $[12,13]$. A promising novel method is OCT. With a ten times greater resolution, it is the ideal tool for studying the microanatomy of a plaque $[14,15]$. Much scientific attention is currently focused on OCT due to its great potential for VP identification [16, 17].

Some diagnostic methods are directed at measuring a plaque's metabolic activity and mechanical properties to predict the risk of its disruption (e.g., intravascular thermography, palpography and elastography). None of these methods have shown much promise thus far [6-8, 18-21].

Recently, intracoronary near-infrared spectroscopy (NIRS) was developed for the determination of the chemical composition of plaques with the intention of identifying lipid cores within lesions [6-8]. Lipid core plaques (LCP) are believed to be clinical correlates of VP [22]. The detection of LCP by NIRS not only provides us with the possibility of identifying VP but also has many other possible applications.

Additionally, a novel catheter system that aims to combine OCT with NIRS to provide an even better characterization of plaques has already been tested on the coronary arteries of human cadavers [25]. The advantages of all above-mentioned methods can be seen in Table I. An overview of the most important studies regarding vulnerable plaque are provided in Table II.

\section{Near-infrared spectroscopy}

Near-infrared spectroscopy is a technique that has been used for decades in the physical sciences to determine the chemical compositions of substances [26]. This method is based on the principle that different substances absorb near-infrared (NIR) light (wavelengths from 800 to 2,500 nm) to different degrees at various wavelengths when the light interacts with certain molecular bonds. Every substance has a characteristic pattern of absorbance that is analogous to a specific NIR fingerprint [26].

This method could obviously be used for the identification of lipid-rich and potentially vulnerable atherosclerotic plaques. The research in this field began in the early 1990s [27], but it took many years to ultimately develop an applicable NIRS catheter. In 2008, Gardner et al. [28] published a prospective study of coronary autopsy specimens that proved that the NIRS-IVUS system is capable of accurately detecting LCP through blood when compared to the gold standard of histological cross-sections [10, 28]. Subsequently, the feasibility of in vivo coronary LCP detection was demonstrated [29]. These findings launched extensive research in the field of NIRS IVUS, and the evidence supporting its capabilities is growing every day. Recently Gardner's results on the accuracy of NIRS to detect a plaque containing a large necrotic core rich in lipids was proven in a large study [30]. The results of the study proved the superiority of NIRS over the analysis of IVUS attenuation [30].

Table I. Characteristic properties of different intravascular imaging methods

\begin{tabular}{|c|c|c|c|c|c|}
\hline Plaque characteristics & OCT & IVUS & NIRS & NIRS-IVUS & RF-IVUS \\
\hline Resolution & $10-20 \mu \mathrm{m}$ & $100 \mu \mathrm{m}$ & & $100 \mu \mathrm{m}$ & $100 \mu \mathrm{m}$ \\
\hline Detection of lipid core & Yes & No & Yes & Yes & Yes \\
\hline Plaque burden assessment & No & Yes & No & Yes & Yes \\
\hline Positive remodelling & No & Yes & No & Yes & Yes \\
\hline Cap thickness & Yes & No & No & No & No \\
\hline Inflammation & Yes & No & No & No & No \\
\hline Calcification & Yes & Yes & No & Yes & Yes \\
\hline Thrombus detection & Yes & Yes & No & Yes & Yes \\
\hline
\end{tabular}




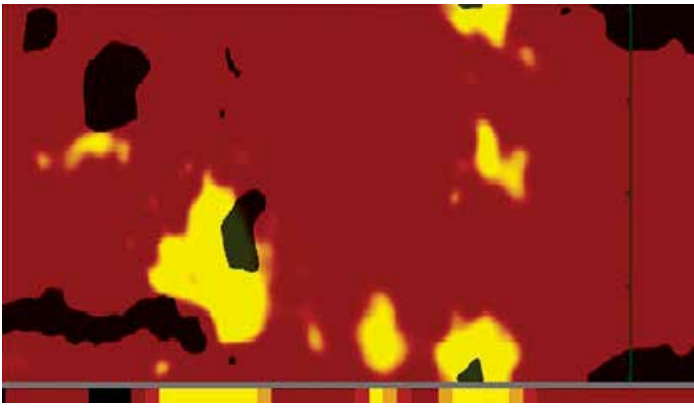

Figure 1. The results of NIRS are presented on a colour-coded probability map called a "chemogram". Every pixel represents the probability of lipid presence at the given location on a colour scale in which low probabilities of lipids are depicted as red, and high probabilities of lipids are shown as yellow. The $X$-axis of the chemogram indicates the pullback position in millimetres, and the $Y$-axis indicates the circumferential position in degrees as though the coronary vessel had been split open along its longitudinal axis

\section{Intravascular near-infrared spectroscopy}

A hybrid catheter that incorporates both NIRS and IVUS (TVC Imaging System, InfraReDx INC. Burlington, MA) is currently available. The combination of NIRS and IVUS in one system has the advantage of producing output that combines both the compositional information provided by NIRS and the structural information provided by IVUS that has the previously proven ability to detect certain properties of a vulnerable atherosclerotic plaque [10, 31].

The results of NIRS are presented on a colour-coded probability map called a "chemogram". Every pixel represents the probability of lipid presence at the given location on a colour scale in which low probabilities of lipids are depicted as red, and high probabilities of lipids are shown as yellow. The $X$-axis of the chemogram indicates the pullback position in millimetres, and the $Y$-axis indicates the circumferential position in degrees as though the coronary vessel had been split open along its longitudinal axis (Figure 1) [32]. Several parameters have been introduced for the quantification of lipid presence in the scanned region. The lipid-core burden index (LCBI) is defined as the fraction of yellow pixels on the chemogram multiplied by 1,000. The maximal lipid core burden index ( $\mathrm{mxLCBI}$ ) per $4 \mathrm{~mm}$ describes the region with the highest lipid burden. Additionally, the mean and maximal angles of the lipid core in the region of interest can be measured to better characterize the lesion's circumferential extent [28, 32-34].

\section{Practical applications of near-infrared spectroscopy}

Although NIRS was specifically developed for the identification of VPs, it seems to have many additional applications in clinical practice. Information about the lipid content of a plaque and the extent of the lipid core in combination with the structural information from IVUS provides clinicians with so-called true vessel characterization. True vessel characterization might aid in the risk stratification of patients prior to treatment for $C A D$, the optimization of percutaneous coronary interventions $(\mathrm{PCl})$, the improvement of stenting and the reduction of the risk of periprocedural myocardial infarction (MI) [35]. The compositional information might also help with the adjustment of pharmaceutical therapy [36].

\section{Prevention of distal embolization}

In parallel with the fact that plaques that contain large lipid pools are prone to spontaneous rupture, these plaques also carry a greater risk of disruption during $\mathrm{PCl}[37,38]$. When such an event occurs, the lipid content of the plaque tends to embolize distally (Figure 2). This results in the obstruction of distal vessels, myocardial injury or periprocedural MI. A relationship between the pre-stenting level of $\mathrm{LCBI}$ and the post-procedural increase in cardiac biomarkers above the threshold for periprocedural MI has been observed in several small studies and case reports [39-43]. Goldstein et al. [42] published data that strongly supported the hypothesis that patients with a plaque with higher lipid content are at a significantly greater risk of periprocedural $\mathrm{MI}$. In this study, $50 \%$ of the patients with $m \times L C B I$ values above the threshold of 500 suffered from periprocedural MI compared to only $4.2 \%$ of the patients with lower $m \times L C B \mid$ $(p<0.001)$ [42]. The above-mentioned findings seem to justify the use of protective devices in patients with high LCBI. This hypothesis was tested in the CANARY trial that was recently presented at the Transcatheter Cardiovascular Therapeutics (TCT) 2014 Congress (Coronary Assessment by Near-infrared of Atherosclerotic Rupture-prone Yellow, NCT01268319). The LCBI values of single native coronary artery lesions in 85 patients undergoing $\mathrm{PCl}$ were prospectively assessed. The study proved that marked reductions in the plaque's lipid contents, as determined by significant decreases in both $\mathrm{LCBI}$ and $\mathrm{mxLCBI}$, followed $\mathrm{PCl}$. The patients who developed periprocedural $\mathrm{MI}$, as defined by at least three-fold elevations in cardiac biomarkers (i.e., cardiac troponin T, cardiac troponin I or creatine kinase-MB) above the normal limit, tended to have higher LCBI. Although this difference did not reach statistical significance in this small sample, the data suggest, in accordance with the above-mentioned trials, that high LCBI may be associated with periprocedural MI. A subgroup of 31 patients with $m \times L C B I \geq 600$ was then randomized to either $\mathrm{PCl}$ with a distal 


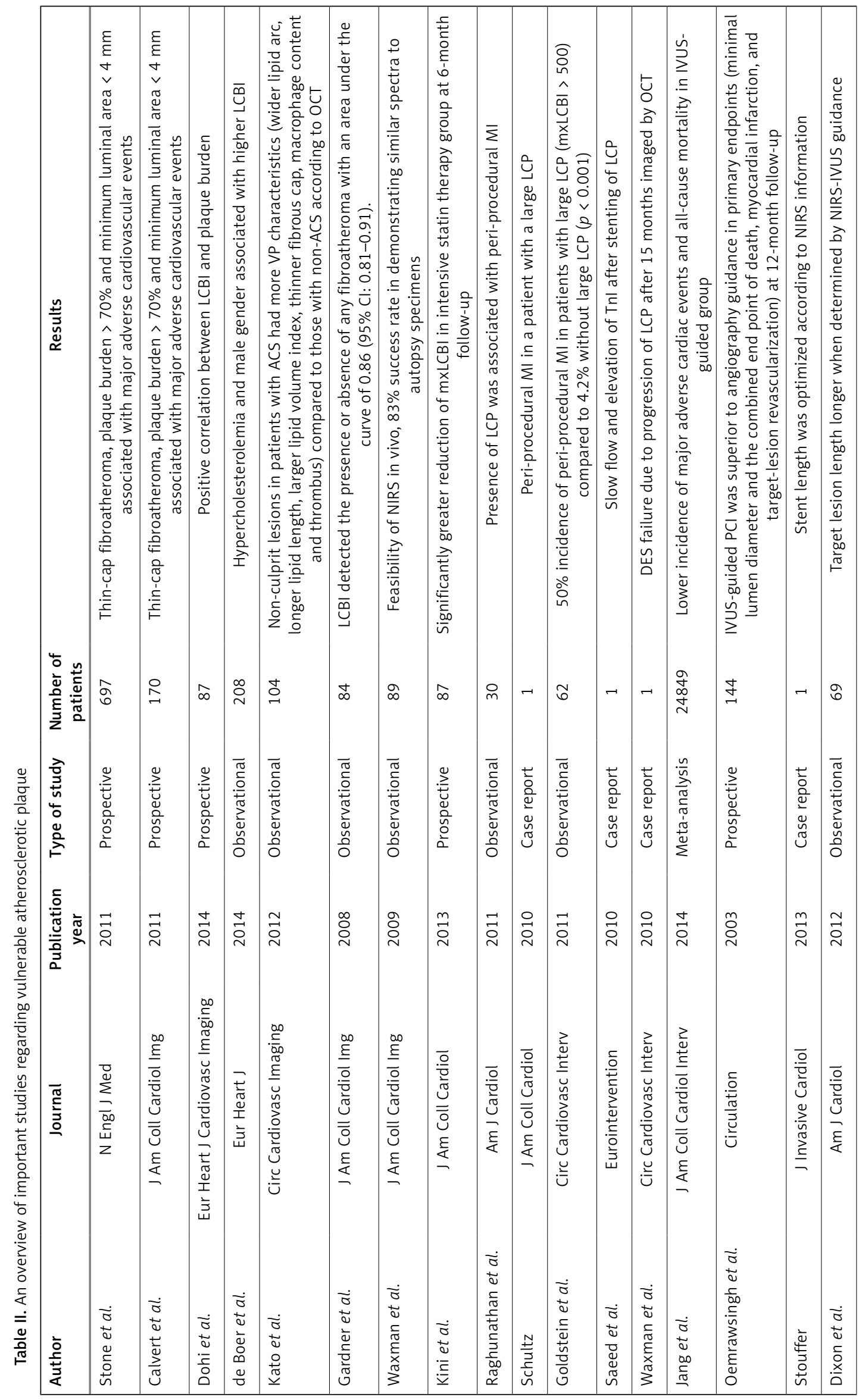




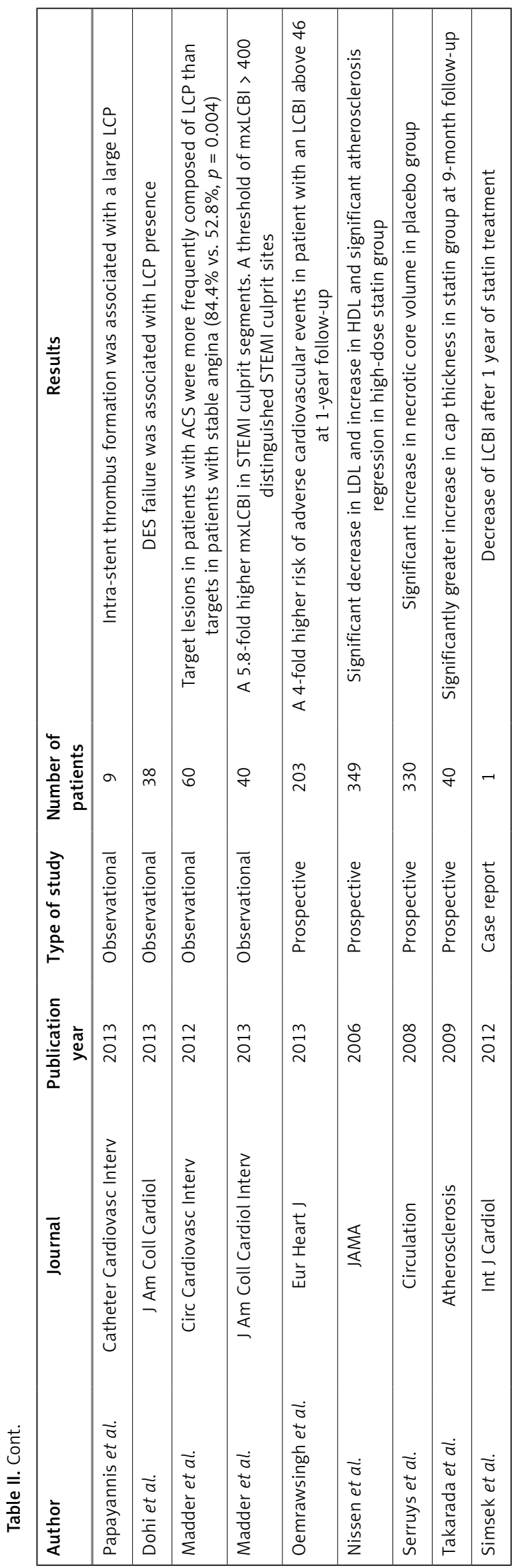

protection filter or $\mathrm{PCl}$ alone. Interestingly, the trial did not show any reduction in the risk of periprocedural MI between these groups. We hypothesize that periprocedural MI was caused by distal embolization of LCP. Since the lipid debris is semi-liquid, a protection filter might not provide sufficient protection against the distal embolization. A different type of protection might be needed. Further research is needed.

\section{Optimization of percutaneous coronary intervention}

Although angiograms are still considered to be the gold standard for the identification of coronary anatomy, angiograms are merely negative images of the vessel lumen [44]. Coronary angiography does not provide thorough information about the vessel wall or about the structure of a plaque that is indicated for $\mathrm{PCl}$. Thus, various imaging modalities have been tested to improve the pre-interventional characterization of lesions. The intention is to prevent procedural complications, such as early and late in-stent thrombosis, dissection, or restenosis [45-50]. In a study by Dixon et al. [51], the NIRS-IVUS system exhibited potential in the optimization of stent length to cover the entire LCP. Furthermore, an association between the extent of LCP pre-stenting as detected with NIRS and thrombus formation post-stenting as determined with OCT has been observed in a small study [52]. It has previously been proposed that the extent of LCP might predict DES failure [53]. These results suggest that NIRS might aid the accurate placement of stents via more precise determination of the plaque's extent. This increased accuracy might help to prevent adverse events such as acute thrombosis or dissection. Obviously, larger studies are needed to support this hypothesis.

\section{Detection of vulnerable plaques}

The main purpose of NIRS is to identify lesion with high lipid contents that indicate probable sites of future coronary events. Although much research remains to be performed, we have already achieved several important milestones on this long journey. The fact that the lesions responsible for ACS more frequently harbour lipid cores has been proven in a study that compared ACS culprit sites with lesions responsible for stable CAD [54]. The same authors subsequently published another study that established characteristic NIRS images of ST-elevation myocardial infarction (STEMI) culprit lesions. These images are characterized by large lipid contents and mxLCBI above a certain threshold [55]. Thus, it seems that we have already identified a possible suspect. The more difficult part of this quest is to prove the suspect guilty, and this goal remains to be accomplished. 


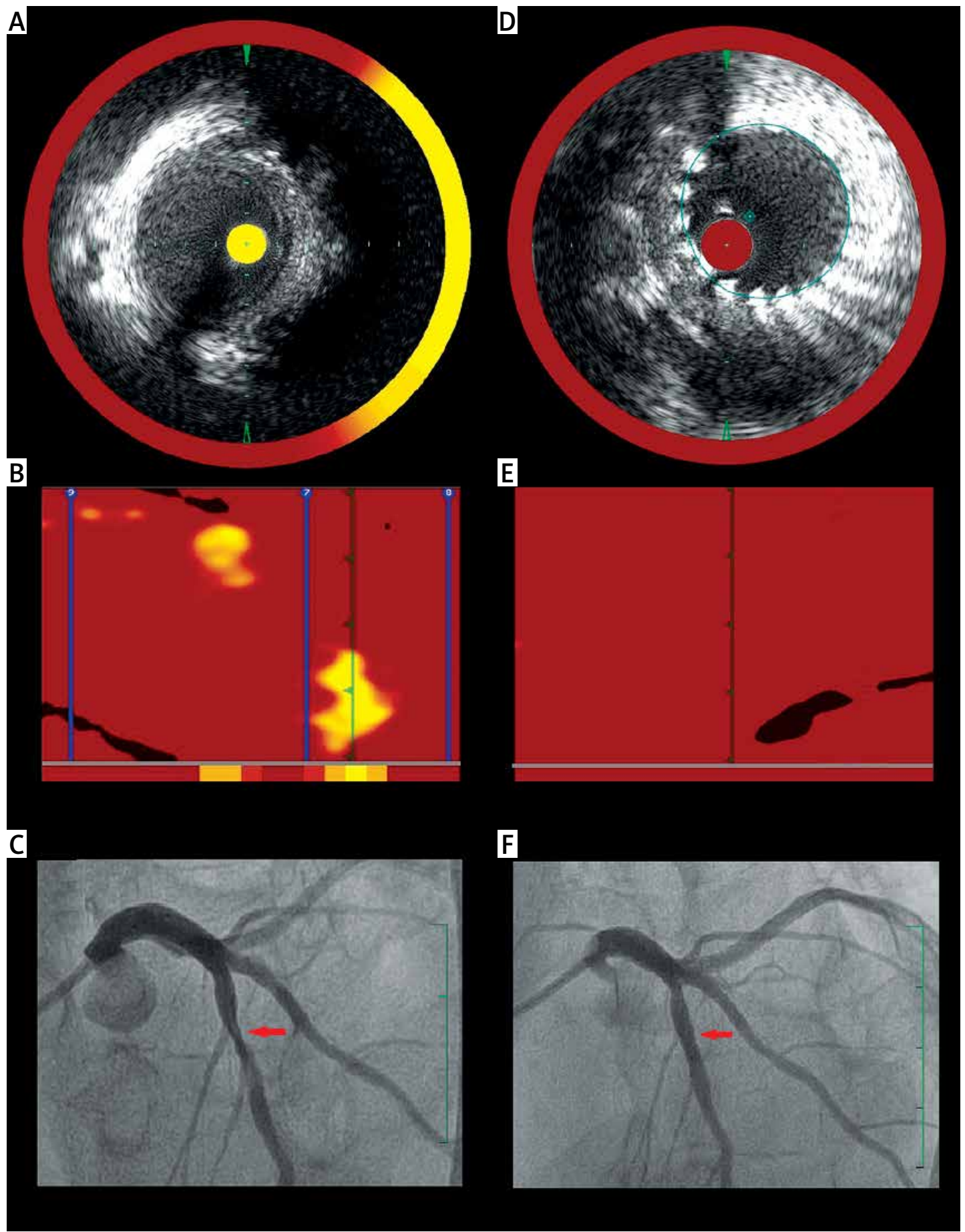

Figure 2. Panel A reveals the intravascular ultrasound (IVUS) image of a lesion in the left anterior descending artery prior to percutaneous coronary intervention (PCI). The near-infrared spectroscopy (NIRS) chemogram obtained before $\mathrm{PCl}$ reveals two lipid cores (B). A coronary angiogram of the lesion is provided (C). A second IVUS image acquired after the $\mathrm{PCl}$ shows good apposition of the stent (D). The second NIRS chemogram documented complete disappearance of the lipid cores during the dilation of the lesion (E). A good angiographic result is shown in panel $F$

To achieve this goal, large clinical trials capable of meeting clinical endpoints are needed. A pilot study recently published by Oemrawsingh et al. [56] evaluated the prognostic value of NIRS. In this prospective observational study, non-culprit vessels of 203 patients with either stable CAD or ACS who were referred for $\mathrm{PCl}$ were examined by NIRS. The primary endpoint of the study (i.e., a composite of all-cause mortality, non-fatal ACS, stroke and unplanned coronary revascularization) occurred 4 times more often in the patients with $L C B I$ values that were equal to or above the me- 
dian value of 43 during a 1-year follow-up. Much larger prospective studies are already in the process of patient recruitment. In the PROSPECT II trial (NCT02171065), 900 ACS patients will undergo three-vessel imaging during $\mathrm{PCl}$ for the initial culprit lesion in a protocol similar to that of the PROSPECT I trial. The difference between the trials is that the NIRS-IVUS catheter will be used instead of simple IVUS in the PROSPECT II trial. With the additional information provided by NIRS, the authors plan to build upon the already known IVUS results (i.e., plaque burden is associated with a higher event rate) and determine the lipid contents of the high-risk sites. The Lipid-Rich Plaque study (LRP) (NCT02033694) is a large, prospective, observational, case-control study that is presently in the process of enrolling 9,000 patients who will undergo $\mathrm{PCl}$. The study's primary endpoint is non-culprit lesion-related major adverse cardiac events (NC-MACE), which are defined by a composite of cardiac death, cardiac arrest, non-fatal $\mathrm{MI}, \mathrm{ACS}$, revascularization by coronary artery bypass graft (CABG) or $\mathrm{PCl}$, and/or rehospitalization for progressive angina related to the non-culprit lesion. The study aims to compare the incidence of NC-MACE between the case (large LCP as defined by NIRS-IVUS) and control (small or no LCP detected by NIRS-IVUS) groups at 2 years of follow-up. The study is expected to yield results in December of 2018.

\section{Stabilisation of a plaque}

Obviously, when we succeed in identifying VP in vivo, some means of stabilising them will be needed to prevent adverse events. While the apparent solution is pharmacotherapy, preventive interventions will probably also be necessary.

Many trials have already studied the effects of various medications on different characteristics of VP. These include studies with IVUS, angioscopy, OCT and even NIRS [35, 57-61]. In the ASTEROID trial, high-dose rosuvastatin (40 mg daily) was found to induce significant reductions in IVUS-defined plaque extents at 2 years of follow-up, although the net reductions were very small [56]. A different study with OCT demonstrated that statin therapy significantly increased the coronary fibrous-cap thickness during 9 months of follow-up in patients with ACS compared to patients who discontinued statin therapy [59]. Changes in NIRS-determined plaque compositions after one year of high-dose statin therapy were described in a case study [60]. In the prospective YELLOW trial, 87 diabetes patients with multivessel CAD, a lesion indicated for $\mathrm{PCl}$ and one other significant stenosis were randomized to aggressive (rosuvastatin $40 \mathrm{mg}$ daily) or standard-of-care lipid-lowering therapy. The non-target lesions were evaluated for lipid content and plaque morphology with NIRS-IVUS during the first $\mathrm{PCl}$ and at 7 weeks of follow-up. Significant changes in both the NIRS and IVUS parameters were observed in the high-dose statin group. These results suggest that statin therapy reduces the lipid contents of lesions and potentially reduces the vulnerability of those lesions [36]. However, this study had some limitations, the most important of which was that the baseline characteristics of the patients in the two randomized arms were considerably different. The results of the better-designed YELLOW II trial (NCT01837823) are expected to be available in March 2015. Importantly, although the changes in the parameters observed in the above-mentioned studies were statistically significant, the absolute changes were very minor. A much larger study will be needed to prove the clinical endpoints.

Whether preventive $\mathrm{PCl}$ of possible VP sites will provide some benefits remains unknown. Presently, we do not have evidence supporting this hypothesis. A large substudy of the PROSPECT II trial called the PROSPECT ABSORB (NCT02171065) seeks to answer this question. In this trial, which is currently recruiting participants, patients with an angiographically and fractional flow reserve (FFR)-insignificant lesion will be randomized to two groups. In one group, the lesions will be treated with a bioresorbable vascular scaffold (BVS) and a guideline-directed medical therapy, and the second group will be treated with medical therapy alone. This study aims to determine whether the BVS safely increases the minimal lumen diameter at 2 years of follow-up.

\section{Conclusions}

In recent years, interventional cardiologists have accepted the fact that improved characterization of coronary atherosclerosis is needed. We have shifted our attention from the simple determination of the degree of arterial stenosis to the study of the anatomy and composition of atherosclerotic plaques. Much progress has been achieved, but the larger portion of the task remains to be completed. Our ultimate goals are the ability to estimate the risk of atherosclerotic disease in a specific patient and to identify vulnerable lesions to tailor coronary heart disease treatment accordingly. Coronary near-infrared spectroscopy paired with intravascular ultrasound appears to be very promising tool to achieve these goals. This diagnostic method has already yielded important evidence that is sufficient to raise our hopes. Spectroscopy seems to have identified a possible correlate of VP in vivo and appears to be useful for the optimization of coronary interventions. It is important to note that the studies 
that have been performed to date have not been sufficiently large to prove any clinical endpoints. Thus, we are still at the very beginning of this research. Large clinical trials are needed to determine whether this method will prove valuable or be condemned to oblivion.

\section{Conflict of interest}

The authors declare no conflict of interest.

\section{References}

1. Go AS, Mozaffarian D, Roger VL, et al. American Heart Association Statistics Committee and Stroke Statistics Subcommittee. Heart disease and stroke statistics-2014 update: a report from the American Heart Association. Circulation 2014; 129: e28-292.

2. Nichols M, Townsend N, Scarborough P, Rayner M. Cardiovascular disease in Europe 2014: epidemiological update. Eur Heart J 2014; 35: 2950-9.

3. Friedman M, Van den Bovenkamp GJ. The pathogenesis of a coronary thrombus. Am J Pathol 1966; 48: 19-44.

4. Muller JE, Tofler GH, Stone PH. Circadian variation and triggers of onset of acute cardiovascular disease. Circulation 1989; 79: 733-43.

5. Virmani R, Burke AP, Farb A, Kolodgie F. Pathology of the vulnerable plaque. J Am Coll Cardiol 2006; 47: C13-8.

6. Virmani R, Kolodgie FD, Burke AP, Farb A, Schwartz SM. Lessons from sudden coronary death: a comprehensive morphological classification scheme for atherosclerotic lesions. Arterioscler Thromb Vasc Biol 2000; 20: $1262-75$.

7. Sanon S, Dao T, Sanon VP, Chilton R. Imaging of vulnerable plaques using near-infrared spectroscopy for risk stratification of atherosclerosis. Curr Atheroscler Rep 2013; 15: 304.

8. Sharif F, Murphy RT. Current status of vulnerable plaque detection. Catheter Cardiovasc Interv 2010; 75: 135-44.

9. Bruggink JL, Meerwaldt R, van Dam GM, et al. Spectroscopy to improve identification of vulnerable plaques in cardiovascular disease. Int J Cardiovasc Imaging 2010; 26: 111-9.

10. Motoyama S, Sarai M, Narula J, Ozaki Y. Coronary CT angiography and high-risk plaque morphology. Cardiovasc Interv Ther 2013; 28: 1-8.

11. Stone GW, Maehara A, Lansky AJ, et al. A prospective natural-history study of coronary atherosclerosis. N Engl J Med 2011; 364: 226-35.

12. Calvert PA, Obaid DR, O'Sullivan M, et al. Association between IVUS findings and adverse outcomes in patients with coronary artery disease: the VIVA (VH-IVUS in Vulnerable Atherosclerosis) Study. J Am Coll Cardiol Img 2011; 4: 894-901.

13. Dohi T, Maehara A, Moreno PR, et al. The relationship among extent of lipid-rich plaque, lesion characteristics, and plaque progression/regression in patients with coronary artery disease: a serial near-infrared spectroscopy and intravascular ultrasound study. Eur Heart J Cardiovasc Imaging 2015; 16: 81-7.

14. de Boer SP, Brugaletta S, Garcia-Garcia HM, et al. Determinants of high cardiovascular risk in relation to plaque-composition of a non-culprit coronary segment visualized by near-infrared spectroscopy in patients undergoing percutaneous coronary intervention. Eur Heart J 2014; 35: 282-9.
15. Jang IK, Tearney GJ, MacNeil B, et al. In vivo characterization of coronary atherosclerotic plaque by use of optical coherence tomography. Circulation 2005; 111: 1551-5.

16. Tearney GJ, Yabushita H, Houser SL, et al. Quantification of macrophage content in atherosclerotic plaques by optical coherence tomography. Circulation 2003; 107: 113-9.

17. Ino Y, Kubo T, Tanaka A, et al. Difference of culprit lesion morphologies between ST-segment elevation myocardial infarction and non-ST-segment elevation acute coronary syndrome: an optical coherence tomography study. JACC Cardiovasc Interv 2011; 4: 76-82.

18. Kato K, Yonetsu T, Kim SJ, et al. Nonculprit plaques in patients with acute coronary syndromes have more vulnerable features compared with those with nonacute coronary syndromes: a 3-vessel optical coherence tomography study. Circ Cardiovasc Imaging 2012; 5: 433-40.

19. Suh WM, Seto AH, Margey RJ, Cruz-Gonzalez I, Jang IK. Intravascular detection of the vulnerable plaque. Circ Cardiovasc Imaging 2011; 4: 169-78.

20. de Korte CL, Carlier SG, Mastik F, et al. Morphological and mechanical information of coronary arteries obtained with intravascular elastography; feasibility study in vivo. Eur Heart J 2002; 23: 405-13.

21. de Korte CL, Pasterkamp G, van der Steen AF, Woutman HA, Bom N. Characterization of plaque components with intravascular ultrasound elastography in human femoral and coronary arteries in vitro. Circulation 2000; 102: 617-23.

22. Schaar JA, de Korte CL, Mastik F, et al. Intravascular palpography for high-risk vulnerable plaque assessment. Herz 2003; 2: 488-95.

23. Fleg JL, Stone GW, Fayad ZA, et al. Detection of highrisk atherosclerotic plaque: report of the NHLBI Working Group on current status and future directions. J Am Coll Cardiol Img 2012; 5: 941-55.

24. Yuehua L, Chenghui Z, Xianliang Z, Lihuan L, Rutai H. Pregnancy-associated plasma protein A predicts adverse vascular events in patients with coronary heart disease: a systematic review and meta-analysis. Arch Med Sci 2013; 9: 389-97.

25. Fard AM, Vacas-Jacques P, Hamidi E, et al. Optical coherence tomography: near infrared spectroscopy system and catheter for intravascular imaging. Opt Express 2013; 21: 30849-58.

26. Caplan JD, Waxman S, Nesto RW, Muller JE. Near-infrared spectroscopy for the detection of vulnerable coronary artery plaques. J Am Coll Cardiol 2006; 47: C92-6.

27. Cassis LA, Lodder RA. Near-IR imaging of atheromas in living arterial tissue. Anal Chem 1993; 65: 1247-56.

28. Gardner CM, Tan H, Hull EL, et al. Detection of lipid core coronary plaques in autopsy specimens with a novel catheter-based near-infrared spectroscopy system. JACC Cardiovasc Imaging 2008; 1: 638-48.

29. Waxman S, Dixon SR, L'Allier P, et al. In vivo validation of a catheter-based near-infrared spectroscopy system for detection of lipid core coronary plaques: initial results of the SPECTACL study. JACC Cardiovasc Imaging 2009; 2: 858-68.

30. Kang S, Mintz GS, Pu J, et al. Combined IVUS and NIRS detection of fibroatheromas: histopathological validation in human coronary arteries. J Am Coll Cardiol Img 2015; 8: 184-94.

31. Rizik D, Goldstein JA. NIRS-IVUS imaging to characterize the composition and structure of coronary plaques. J Invasive Cardiol 2013; 25: 2A-4A. 
32. Pu J, Mintz GS, Brilakis ES, et al. In vivo characterization of coronary plaques: novel findings from comparing greyscale and virtual histology intravascular ultrasound and near-infrared spectroscopy. Eur Heart J 2012; 33: 372-83.

33. Horváth M, Hájek P, Štěchovsky C, Veselka J. Vulnerable plaque imaging and acute coronary syndrome. Cor Vasa 2014; 56: e362-8.

34. Štěchovský C, Horváth M, Hájek P, Veselka J. Detection of vulnerable atherosclerotic plaque with near-infrared spectroscopy: a systematic review. Vnitr Lek 2014; 60: 375-9.

35. Brugaletta S, Garcia-Garcia HM, Serruys PW. Perspective on the use of true vessel characterization imaging in interventional cardiology clinical practice. Interv Cardiol 2012; 7: 17-20.

36. Kini AS, Baber U, Kovacic JC, et al. Changes in plaque lipid content after short-term intensive versus standard statin therapy: the YELLOW trial (reduction in yellow plaque by aggressive lipid-lowering therapy). J Am Coll Cardiol 2013; 62: 21-9.

37. Niccoli G, Burzotta F, Galiuto L, Crea F. Myocardial no-reflow in humans. J Am Coll Cardiol 2009; 54: 281-92.

38. Prasad A, Herrmann J. Myocardial infarction due to percutaneous coronary intervention. N Engl J Med 2011; 364: 453-64.

39. Raghunathan D, Abdel-Karim AR, Papayannis AC, et al. Relation between the presence and extent of coronary lipid core plaques detected by near-infrared spectroscopy with postpercutaneous coronary intervention myocardial infarction. Am J Cardiol 2011; 107: 1613-8.

40. Schultz CJ, Serruys PW, van der Ent M, et al. First-in-man clinical use of combined near-infrared spectroscopy and intravascular ultrasound: a potential key to predict distal embolization and no-reflow? J Am Coll Cardiol 2010; 56: 314.

41. Goldstein JA, Maini B, Dixon SR, et al. Detection of lipid-core plaques by intracoronary near-infrared spectroscopy identifies high risk of periprocedural myocardial infarction. Circ Cardiovasc Interv 2011; 4: 429-37.

42. Goldstein JA, Grines C, Fischell T, et al. Coronary embolization following balloon dilation of lipid-core plaques. JACC Cardiol Imaging 2009; 2: 1420-4.

43. Saeed B, Banerjee S, Brilakis ES. Slow flow after stenting of a coronary lesion with a large lipid core plaque detected by near-infrared spectroscopy. Eurolntervention 2010; 6: 545.

44. Topol EJ, Nissen SE. Our preoccupation with coronary luminology. The dissociation between clinical and angiographic findings in ischemic heart disease. Circulation 1995; 92: 2333-42.

45. Sakhuja R, Suh WM, Jaffer FA, Jang IK. Residual thrombogenic substrate after rupture of a lipid-rich plaque: Possible mechanism of acute stent thrombosis? Circulation 2010; 122: 2349-50.

46. Waxman S, Freilich MI, Suter MJ, et al. A case of lipid core plaque progression and rupture at the edge of a coronary stent: elucidating the mechanisms of drug-eluting stent failure. Circ Cardiovasc Interv 2010; 3: 193-6.

47. Jang JS, Song YJ, Kang W, et al. Intravascular ultrasound-guided implantation of drug-eluting stents to improve outcome: a meta-analysis. JACC Cardiovasc Interv 2014; 7 : 233-43.

48. Oemrawsingh PV, Mintz GS, Schalij MJ, Zwinderman $A H$, Jukema JW, van der Wall EE. Intravascular ultrasound guidance improves angiographic and clinical outcome of stent implantation for long coronary artery stenoses: final results of a randomized comparison with angio- graphic guidance (TULIP Study). Circulation 2003; 107: 62-7.

49. Lee CW, Kang SJ, Park DW, et al. Intravascular ultrasound findings in patients with very late stent thrombosis after either drug-eluting or bare-metal stent implantation. J Am Coll Cardiol 2010; 55: 1936-42.

50. Guagliumi G, Sirbu V, Musumeci G, et al. Examination of the in vivo mechanisms of late drug-eluting stent thrombosis: findings from optical coherence tomography and intravascular ultrasound imaging. JACC Cardiovasc Interv 2012; 5: 12-20.

51. Dixon SR, Grines CL, Munir A, et al. Analysis of target lesion length before coronary artery stenting using angiography and near-infrared spectroscopy versus angiography alone. Am J Cardiol 2012; 109: 60-6.

52. Papayannis AC, Abdel-Karim AR, Mahmood A, et al. Association of coronary lipid core plaque with intrastent thrombus formation: a near-infrared spectroscopy and optical coherence tomography study. Catheter Cardiovasc Interv 2013; 81: 488-93.

53. Dohi T, Weisz G, Powers ER, et al. TCT-583 The extent of lipid-rich plaque assessed by near-infrared spectroscopy may predict DES failure: a COLOR Registry Analysis. J Am Coll Cardiol 2013; 62: B176-7.

54. Madder RD, Smith JL, Dixon SR, Goldstein JA. Composition of target lesions by near-infrared spectroscopy in patients with acute coronary syndrome versus stable angina. Circ Cardiovasc Interv 2012; 5: 55-61.

55. Madder RD, Goldstein JA, Madden SP, et al. Detection by near-infrared spectroscopy of large lipid core plaques at culprit sites in patients with acute ST-segment elevation myocardial infarction. JACC Cardiovasc Interv 2013; 6: $838-46$

56. Oemrawsingh RM, Cheng JM, García-García HM, et al. Near-infrared spectroscopy predicts cardiovascular outcome in patients with coronary artery disease. J Am Coll Cardiol 2014; 64: 2510-8.

57. Nissen SE, Nicholls SJ, Sipahi I, et al. Effect of very high-intensity statin therapy on regression of coronary atherosclerosis: the ASTEROID trial. JAMA 2006; 295: 1556-65.

58. Serruys PW, García-García HM, Buszman P, et al. Integrated Biomarker and Imaging Study-2 Investigators. Effects of the direct lipoprotein-associated phospholipase $A(2)$ inhibitor darapladib on human coronary atherosclerotic plaque. Circulation 2008; 118: 1172-82.

59. Takarada S, Imanishi T, Kubo T, et al. Effect of statin therapy on coronary fibrous-cap thickness in patients with acute coronary syndrome: assessment by optical coherence tomography study. Atherosclerosis 2009; 202: 491-7.

60. Simsek C, van Geuns RJ, Magro M, Boersma E, GarciaGarcia HM, Serruys PW. Change in near-infrared spectroscopy of a coronary artery after 1-year treatment with high dose rosuvastatin. Int J Cardiol 2012; 157: e54-6.

61. Banach M, Serban C, Sahebkar A, et al. Impact of statin therapy on coronary plaque composition: a systematic review and meta-analysis of virtual histology-intravascular ultrasound studies. BMC Med 2015; 13: 229. 